\title{
Different Stages of Evolution of Prominence and the Associated CMEs
}

\author{
Lela Taliashvili ${ }^{1}$, Zadig Mouradian ${ }^{2}$ and Heidy Gutiérrez ${ }^{1}$ \\ ${ }^{1}$ Space Research Center, University of Costa Rica, 2060 San Jos, Costa Rica \\ email: lela.taliashvili@cinespa.ucr.ac.cr \\ ${ }^{2}$ Observatoire de Paris-Meudon, LESIA, 92190 Meudon, France
}

\begin{abstract}
We study the different evolutional stages of a large quiescent prominence, mainly considering its dynamic/thermal instabilities occurred close from the boundary of coronal hole $(\mathrm{CH})$. We identify the critical conditions, such as the minimum distance between the CH's boundary and prominence channel and the emergence of a new magnetic flux linked to the prominence instability and its general evolution in connection to $\mathrm{CH}$. Our observations indicate peculiar filament activations prior to its thermal/dynamic instabilities, suggesting the connection of nearby $\mathrm{CH}$ with the general evolution of prominence and vice versa. Additionally, we analyze each evolutional stage of prominence and the associated Coronal Mass Ejections (CMEs).
\end{abstract}

Keywords. Prominence, coronal hole, coronal mass ejection, magnetic flux, magnetic field

\section{Introduction}

An important issue for understanding the evolution of prominences/filaments is their thermal and dynamic instabilities (Mouradian et al. (1981), Mouradian et al. (1986), Tandberg-Hanssen (1995)). Observational results indicate the implication of $\mathrm{CH}$ at these different evolutional stages of prominence. Based on 42 quiescent prominences, the study by Taliashvili et al. (2009) have found that all thermal and $91 \%$ of dynamic eruptions were occurred within $\sim 15^{\circ}$ distance from CH's boundary; and $47 \%$ of thermal and $64 \%$ of dynamic eruptions were associated with CMEs. It is well known the relation between the prominence dynamic eruptions and CMEs due to magnetic reconnection, frequently anticipated by an emergence of new magnetic flux at one of filament's foot points (Mouradian et al. (1987)) while the energy is transported by waves along the flux tubes in the prominence feet from the pivot point (Mouradian et al. (1989)). Various authors have discussed different tools associated to the magnetic reconfiguration before/after prominence eruption and the formation of CMEs; Schmieder et al. (2013) (and their references) summarizing the causes of prominence eruption, the emergence of new flux and/or the external magnetic field dispersion and/or reconnection of field lines below/above the flux rope that reduces the downward magnetic tension, brings the flux rope to an unstable state; however they conclude that the most efficient mechanism for CME formation is torus instability. Yet these processes are still unclear step by step.

\section{Event description and Discussion}

We study the continuous evolution of a long-lived quiescent filament / prominence spread out from equator to $-19^{\circ}$ during two periods, Aug. 3-13 (CR 2126), Aug. 30Sept. 4 of 2012 (CR 2127), located at $\sim 15^{\circ}$ distance from the equatorial $\mathrm{CH}$; based on $\mathrm{H} \alpha$ images reported by Paris-Meudon Observatory and GONG. In order to study the evolutional stages of filament and the global interaction with $\mathrm{CH}$ evolution, we analyze: 


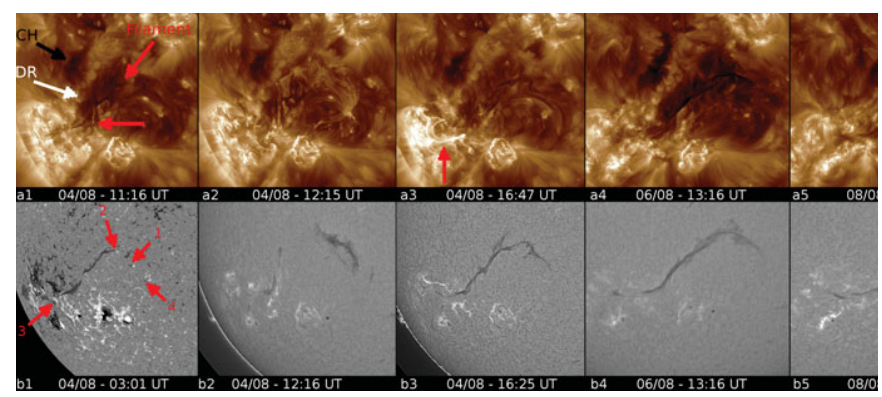

Figure 1. (a1-a6) EUV evolution of filament/CHs by SDO/AIA-193 A. (b2-b6) H $\alpha$ evolution of filament by GONG. (b1) Superposition of GONG H $\alpha$ image and SDO/HMI magnetogram of 04/08-03:00UT, with four newly emerged fluxes.

STEREO and SDO/AIA EUV images, WSO, SDO/HMI magnetograms, and maps. In regard to the prominence eruption-CME association we consider their similar position angles, widths and their starting times. CMEs are identified using SOHO/LASCO and STEREO CME catalogs, images and movies.

The large $\left(\sim 45^{\circ}\right)$ filament has an additional northern unstable $\sim 15^{\circ}$ section, extended toward the flux 4 (Figure 1, b1), which is observed only at EUV images, showing that the prominence plasma reached coronal temperatures, while keeping its shape i.e. the magnetic field structure. Four small active regions (ARs) surround the southern foot point of filament. At the East side, parallel to the filament channel extends the dimming region (DR) and a small equatorial $\mathrm{CH}$ within $\sim 15^{\circ}$ distance (Figure 1, a1). Every evolutional stage of filament successively involves three sections that compose the filament's body: southern (SS), central (CS) and northern (NS); each of them characterized by a different evolution Mouradian et al. (1989) and an independent and almost constant movement of its material that sometimes upraises, but the whole filament maintains stable until Aug. 8. During the evolution of this filament, additionally three peculiar motions are observed at Aug. 4, 6 and 7 in form of a directional plasma launch. These motions anticipate a consecutive emergence of certain new magnetic fluxes near and along the filament channel, especially close to the filament foot points (Figure 1, b1; additional fluxes emerge among flux 2/3, at the east side of filament channel). Moreover, C1.1-C3.5 flares, related with NOAA ARs 11539-40-41 observed $0.5 \mathrm{~h}-3 \mathrm{~h}$ before these launches. Then a directional plasma launch starts from southern toward the northern foot point that follows the same path of emerged fluxes ending with filament appearance at its original position. These processes develop according to the following three scenarios (Figure 1): (1) Directional plasma launch (Aug. 4. H $\alpha$ : 11:15-13:30 UT; EUV: 11:15-18:00 UT) from a small EUV loop that reconnects with the southern foot point at 11:16 UT; At 13:30 UT filament returns to its original form in $\mathrm{H} \alpha$ due to plasma cooling by radiation; then it raises and its SC expands due to an increasing energy input leading to its thermal disappearance in $\mathrm{H} \alpha$ (13:45-14:45 UT); At 15:40 flux 3 cancelled and a small coronal loop arise from its location, at 16:40 UT, moving radially; the northern EUV hot section cools down; this NS that was visible only at EUV, appears in $\mathrm{H} \alpha$ too. After $\sim 4 \mathrm{~h}$, two consecutive narrow CMEs start at nearly position angle of the cancelled flux; after CMEs, filament and $\mathrm{CH}$ increase, reaching their maximum size at Aug. 6-7. (2) Directional plasma launch (Aug. 6, 09:15-22:45 UT) combined with filament plasma motions mainly inside its sections, accompanied by the whole filament expansion and the transient $\mathrm{CH}$ birth within DR, associated with evacuated filament plasma (20:45 UT); transient $\mathrm{CH}$ grows and starts the CS and SS thermal disappearance. (3) Directional plasma launch (Aug. 7, 23:01 UTAug. 8. 02:00 UT) is similar to the first launch; nonetheless is followed by a dynamic 
eruption of CS and SS (03:45 UT) and perturbation of filament's surroundings, covering the $\mathrm{CH}$; after $\sim 30 \mathrm{~min}$. two consecutive $\mathrm{CMEs}$ start and both $\mathrm{CHs}$ disappear (14:02 UT). At Aug. 13, the SS and the CS's remnant erupt followed by consecutive CMEs (two west-limb and one Halo). Based on STEREO EUV evolution of filament we note the filament rebuild (Mouradian et al. 1987) from Aug. 14, almost with its original form. Lately, from Aug. 30 (CR 20127) it is observed again from Earth with two DRs, at east side (within $\sim 15^{\circ}$ distance) and around its NS. At 17:00 UT, Aug. 31 the whole filament eruption begins and at 20:00 UT a Halo CME occurs. A new small equatorial $\mathrm{CH}$ is formed within DR at the east side of filament channel few hours before this eruption, which grows during the eruption and disappears slowly after 3.5 days. We observed a part of the evacuated filament's material right after this eruption. From Sept. 4, H $\alpha$ filament is observed entirely with considerably less material.

As a summary of our observations, we interpret that new $\mathrm{CH}$ appears before and grows during the thermal/dynamic instabilities, whereas $\mathrm{CH}$ fading is a consequence of dynamic instabilities or/and after classical CMEs (Taliashvili et al. (2009)); we observe the $\mathrm{CH}$ growth only after CMEs associated with thermal instabilities. A stable magnetic filed supports the filament. Thermal instabilities and the associated CMEs prior to the filament eruption are related to a small-scale magnetic reconfiguration, but they might be indicators of upcoming destabilization of filament and its surroundings (including adjacent $\mathrm{CH}$, within $15^{\circ}$ distance from the filament channel) followed by a huge CMEs and a large-scale magnetic reconfiguration observed at the late evolutional stage of filament as longitudinal magnetic diffusion (Gutiérrez et al. (2013)), with CH fading from the closest region to the southern foot point toward the $\mathrm{CH}$. These observational results indicate the involvement of a newly emerged magnetic flux near/along of filament channel and close to the foot points at the starting process of instabilities and also at the general evolution of filament/adjacent $\mathrm{CH}$; ongoing series of magnetic reconnections due to flux cancelation leading to peculiar motions and different type of filament instabilities, birth and growth of adjacent $\mathrm{CH}$ and formation of CMEs. The flux rope that is progressively formed by photospheric reconnection and successively flux cancellation, approaches a critical point of the equilibrium curve driven by a constant increase of the twist and/or by changing the magnetic flux below/above the flux rope, removes the overlying arcades by coronal reconnection and erupts by developed torus instability (Aulanier et al. (2010) and their references).

Acknowledgments. We thank the Observatoire Paris-Meudon, GONG, WSO, SDO, SOHO/LASCO, STEREO for open access to their data sets. L.T and H.G. acknowledge for the financial supports by IAU/LOC.

\section{References}

Aulanier, G., Török, T., Démoulin, P., \& DeLuca, E. E. Astrophys. J. 708, 314, 2010.

Gutiérrez, H., Taliashvili, L., \& Mouradian, Z. Ad. Space Res. 51, 1824, 2013.

Mouradian, Z., Martres, M. J., \& Soru-Escaut, I. Proc. Japan-France Seminar on Solar Physics, Tokyo, ed., Moriyama, F., and Henoux, J.C. 195, 1981.

Mouradian, Z., Martres, M. J., \& Soru-Escaut, I. Coronal and Prominence Plasmas, NASA conf. Public. ed., Poland A. I. 221, 1986.

Mouradian, Z., Martres, M. J., Soru-Escaut, I., \& Gesztelyi, L. Astron. Astrophys. 183, 129, 1987.

Mouradian, Z., \& Soru-Escaut, I. Astron. Astrophys. 210, 410, 1989.

Schmieder, B., Dmoulin, P., \& Aulanier, G. Adv. Space Res 51, 1967, 2013.

Tandberg-Hanssen, E. The Nature of Solar Prominences, Kluwer Acad. Pub. §6.2.1, 1995.

Taliashvili, L., Mouradian, Z., \& Páez, J. Sol. Phys. 258, 277, 2009. 